\title{
Modeling, Understanding, and Interacting with Humans
}

\author{
Yoshihiko Nakamura \\ University of Tokyo \\ Department of Mechano-Informatics and \\ Information and Robotics Technology Research Initiative \\ nakamura@ynl.t.u-tokyo.ac.jp \\ http://www.ynl.t.u-tokyo.ac.jp
}
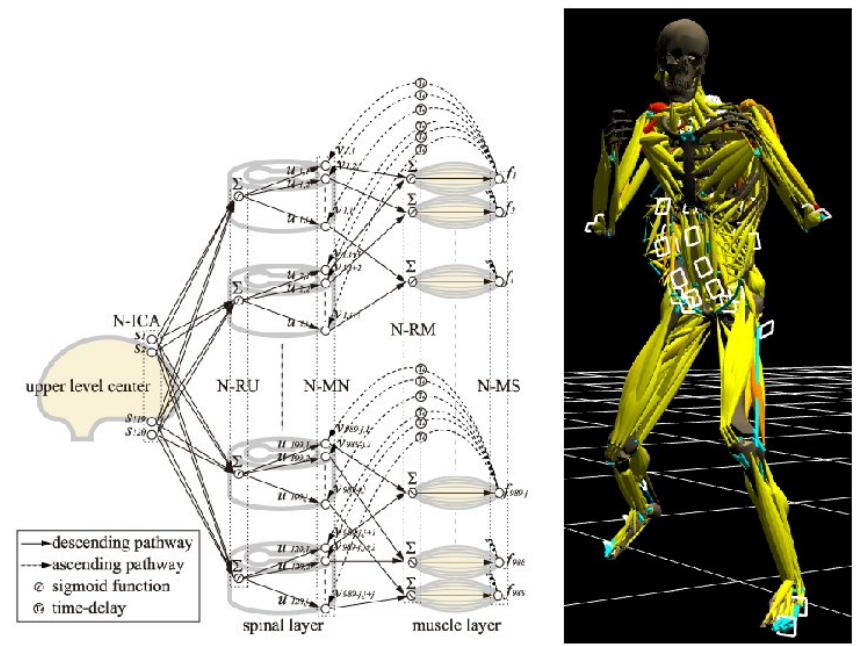

Machines and robots extend their frequency and quality of interaction with humans. Tools invented by humans have shown evolution in the history. One may find a similar genealogical tree of tools to the evolution of life. Machines that interact with humans based on understanding humans are in a sense the ultimate tools for humans. The advance of computational algorithms and modeling technology in robotics encourages us making a challenge pursuing such machines. My talk will highlight and introduce our recent research results on emulating somatosensory sensation of humans, semiotics of human whole-body motion patterns, and using them for machines interacting with humans. 